\title{
Desempenho Silvicultural de Jacaranda copaia (Aubl.) D. Don sob Diferentes Espaçamentos no Oeste do Estado do Pará
}

\author{
Performance Silvicultural Jacaranda copaia (Aubl.) D. Don Spacings \\ Under Different in the West of Pará State
}

\author{
Arllen Élida Aguiar Paumgartten', Alberto Bentes Brasil Neto', \\ Vanessa Gomes de Sousa ${ }^{3}$, Silvio Brienza Junior ${ }^{3}$, Jorge Alberto Gazel Yared ${ }^{4}$
}

\begin{abstract}
Resumo:
O objetivo desse estudo foi avaliar o desempenho silvicultural da espécie amazônica Jacaranda copaia em plantio homogêneo, sob diferentes espaçamentos entre plantas em diferentes idades, no Oeste do Estado do Pará. Foram instalados no Campo Experimental da Embrapa Amazônia Oriental no município de Belterra, Oeste do Pará, quatro tratamentos, referentes aos diferentes espaçamentos entre plantas: $3 m \times 2 m ; 3 m \times 3 m ; 3 m \times 4 m$ e $4 m \times 4 m$. As variáveis utilizadas para avaliar o desempenho silvicultural foram: sobrevivência, altura total, diâmetro à altura do peito, área basal, volume por árvore e volume por unidade de área. O povoamento de Jacaranda copaia apresentou elevada sobrevivência independentemente da densidade populacional, que não influenciou o desempenho nessa variável e em altura total. No entanto, para a variável diâmetro houve aumento significativo à medida que o espaço entre plantas era ampliado. A maior densidade de indivíduos promoveu a maior produção em área basal, volume de madeira por hectare e incremento volumétrico. O desempenho silvicultural da espécie nos espaçamentos $3 \mathrm{~m} \times 3 \mathrm{~m}$ e $3 \mathrm{~m} \times 4 \mathrm{~m}$ apresentou semelhança na maioria das variáveis e idades estudadas, com bom desempenho em área basal e Incremento Médio Anual.
\end{abstract}

Palavras-chave: espécie amazônica, reflorestamento, densidade de plantio.

\begin{abstract}
:
The aim of this study was to evaluate the silvicultural performance of the Jacaranda copaia in homogeneous plantation, under different spacing between plants at different ages, in the West of the State of Pará. They were installed in the Experimental Field of Embrapa Amazônia Oriental in the municipality of Belterra, Pará, four treatments, referring to the different spacings between plants: $3 \mathrm{~m} \times 2 \mathrm{~m} ; 3 \mathrm{~m} \times 3 \mathrm{~m} ; 3 \mathrm{~m} \times 4 \mathrm{~m}$ and $4 \mathrm{~m} \times 4 \mathrm{~m}$. The variables used to evaluate the silvicultural performance were: survival, total height, diameter at breast height, basal area, volume per tree and volume per unit area. The Jacaranda copaia stand presented high survival and the population density did not influence the performance in this variable and in total height, however for the variable diameter there was a significant increase as the space between plants was enlarged. The higher density of individuals promoted the higher production in basal area, wood volume per hectare and volumetric increase. The silvicultural performance of the species at $3 \mathrm{~m} \times 3 \mathrm{~m}$ and $3 \mathrm{~m} \times 4 \mathrm{~m}$ spacings showed similarity in most variables and ages studied, with good performance in basal area and Average Annual Increase.
\end{abstract}

Keywords: amazonian species, reforestation, planting density.

\section{INTRODUÇÃO}

Na Amazônia o abastecimento do mercado de produtos florestais provém predominantemente do manejo de florestas naturais. $\mathrm{O}$ avanço das leis que disciplinam a exploração dessas florestas nos últimos anos e a demanda por produtos procedentes de atividades sustentáveis tem incentivado o plantio de espécies florestais nativas para fins comerciais na região (SILVEIRA, 2014).

\footnotetext{
1. Instituto Federal do Pará - IFPA. Breves / PA, Brasil. E-mail: arllen.aguiar@ifpa.edu.br

2. Instituto Federal do Pará - IFPA. Óbidos / PA, Brasil.

3. Empresa Brasileira de Pesquisa Agropecuária - Embrapa Amazônia Oriental. Belém / PA, Brasil.

3. Empresa Brasileira de Pesquisa Agropecuária - Embrapa Amazônia Oriental. Belém / PA, Brasil.

4. Empresa Brasileira de Pesquisa Agropecuária - Embrapa Amapá. Macapá / AP, Brasil.
}

Sci. For., Piracicaba, v. 46, n. 120, p. 701-707, Dez. 2018 DOI: dx.doi.org/10.18671/scifor.v46n120.18 
A utilização de espécies nativas da Amazônia em projetos de reflorestamento depende de informações técnicas sobre os aspectos que influenciam no seu padrão de crescimento e produção. Os estudos existentes abrangem, geralmente, características botânicas e dendrológicas, fazendo com que muitos empresários e produtores não invistam em experiências silviculturais com essas espécies (NASCIMENTO et al., 2012).

Na tentativa de contornar este problema, diversas instituições de pesquisa vêm investigando a potencialidade de espécies nativas da Amazônia para serem usadas em plantios comerciais. Dentre as espécies promissoras está a Jacaranda copaia (Aubl.), pertencente à família botânica Bignoniaceae, comumente chamada na Região Norte de parapará.

O potencial dessa espécie para utilização em plantios comerciais é devido ao rápido crescimento com fuste retilíneo e cilíndrico, à facilidade de propagação de mudas e à possibilidade de utilização da madeira na indústria de compensados, laminados e marcenaria (IPEF, 2016; TONINI et al., 2008). No entanto, ainda são necessárias outras informações silviculturais da espécie, dentre as quais se destaca o espaçamento entre árvores.

A adoção de espaçamento entre árvores inadequado na implantação do povoamento florestal pode influenciar negativamente o desenvolvimento e a produtividade do mesmo. A densidade de plantio influencia a taxa de mortalidade, o crescimento das árvores, o volume e a qualidade da madeira, a idade de rotação silvicultural e outras variáveis que concomitantemente afetam os custos do povoamento (VENTURIN et al., 2014; SCHNEIDER et al., 2015).

Como o espaçamento de plantio é uma característica relevante na tomada de decisão durante a implantação de povoamentos florestais, nesse estudo objetivou-se avaliar o desempenho silvicultural da espécie amazônica Jacaranda copaia em plantio homogêneo, sob diferentes espaçamentos entre plantas em diferentes idades, no Oeste do Estado do Pará.

\section{MATERIAL E MÉTODOS}

O estudo foi realizado em área do campo experimental da Empresa Brasileira de Pesquisas Agropecuária - EMBRAPA, localizada entre as coordenadas geográficas $02^{\circ} 25^{\prime}$ e $03^{\circ} 00^{\prime}$ de latitude sul e de $54^{\circ} 00^{\prime}$ e $55^{\circ} 00^{\prime}$ de longitude a oeste, no Município de Belterra, pertencente à região Oeste do estado do Pará. A região possui um clima do tipo Ami, segundo a classificação de Koppen. A temperatura média anual é de $26,3^{\circ} \mathrm{C}$, umidade relativa do ar em volta de $84 \%$ e a precipitação média anual de $2.077 \mathrm{~mm}$, com o período chuvoso entre os meses de dezembro a junho (Oliveira Júnior \& Correa, 2001).

O experimento foi instalado em uma área de 4,3ha sob um Latossolo Amarelo distrófico de relevo plano e textura muito argilosa. De acordo com Silva et al., (2000) a área experimental apresenta teores de argila acima de $600 \mathrm{~g} \mathrm{~kg}^{-1}$; valores de $\mathrm{pH}$ em água entre 3,7 a 4,9; soma de bases trocáveis muito baixa (0,1 a 1,12 $\mathrm{cmol} \mathrm{kg}^{-1}$ de solo); baixa capacidade de troca efetiva (1,1 a $15 \mathrm{cmol} \mathrm{kg}^{-1} \mathrm{de}^{-}$ solo); saturação por alumínio superior a 50\% e baixa saturação de bases (de 1 a 18\%).

No preparo da área foi efetuada a passagem de "rolo faca" sobre a vegetação (gramíneas do gênero Brachiaria sp. e floresta secundária pouco densa), queimada na leira, gradagem superficial e posteriormente foram demarcadas as parcelas e promovida a abertura de covas com adubação de $330 \mathrm{~g}$ de NPK por cova na formulação (15-25-12).

Foram instalados quatro tratamentos, referentes aos diferentes espaçamentos entre plantas: $3 \mathrm{~m} \times 2 \mathrm{~m} ; 3 \mathrm{~m} \times 3 \mathrm{~m} ; 3 \mathrm{~m} \times 4 \mathrm{~m}$ e $4 \mathrm{~m} \times 4 \mathrm{~m}$. Para o plantio foram utilizadas mudas produzidas no viveiro da EMBRAPA, a partir de sementes provenientes de árvores matrizes do município de Belterra. O plantio foi realizado no mês de julho de 1981.

Durante os dois primeiros anos foram realizadas roçagens manuais no espaçamento $3 \mathrm{~m} \times 2 \mathrm{me}$ mecânica nos demais espaçamentos, duas vezes por ano em toda área. O coroamento foi realizado quatro vezes ao ano somente nas proximidades das plantas. A partir do ano de 1982, foram feitas medições anuais das variáveis: diâmetro à altura do peito (DAP), altura (H) e sobrevivência (S), as quais foram medidas até o ano de 1992.

O desempenho silvicultural das árvores de Jacaranda copaia foi avaliado através da análise das seguintes variáveis: sobrevivência $(S)$ em porcentagem; altura total $(\mathrm{Ht})$ em metros; diâmetro à altura do peito (DAP) em centímetros; área basal (G) $\mathrm{em} \mathrm{m}^{2}$ ha ${ }^{-1}$, estimados pela equação $G=\sum g^{*} F P$, onde 
$g$ é a área transversal $\left(\mathrm{m}^{2}\right)$, a qual foi obtida pela expressão: $g=\varpi^{*} D A P 2 / 40000$ e FP consiste no fator de proporcionalidade de área, razão entre área útil da parcela e o valor de 1 ha $\left(10000 \mathrm{~m}^{2}\right)$.

$\mathrm{O}$ volume por árvore - $\mathrm{v}_{\text {árv }}\left(\mathrm{m}^{3}\right.$ árv $\left.{ }^{1}\right)$ foi obtido pela expressão: $v_{\text {árvore }}=g^{*} H t^{*} f f$; onde ff consiste no fator de forma, no qual adotou-se o 0,5 por ser o valor encontrado para outras espécies amazônicas (TONINI et al., 2005). O volume por unidade de área $-V_{h a}\left(\mathrm{~m}^{3} \mathrm{ha}^{-1}\right)$ foi obtido pela equação: $V h a=\sum V_{\text {árvore }}{ }^{*} F P$. Por sua vez, o incremento médio anual em volume - IMA $\left(\mathrm{m}^{3} \mathrm{ha}^{-1}\right)$ foi estimado pela equação $I M A=V_{\text {árvore }} /$ idade (anos).

Os dados foram submetidos à análise de variância, em delineamento experimental em blocos ao acaso, com quatro tratamentos e quatro repetições, e ao teste de Scott Knott para discriminação das médias dos tratamentos, a 5\% de significância. As análises estatísticas foram efetuadas com o auxílio do programa Sisvar 5.6 (FERREIRA, 2014).

\section{RESULTADOS E DISCUSSÃO}

A sobrevivência não foi influenciada pelos espaçamentos de plantio durante os dez anos de avaliação e até o sétimo ano foi superior a 90\% (Figura 1). Povoamentos com sobrevivência acima de $70 \%$ são considerados de alta sobrevivência segundo a classificação de Carvalho (1982). Com isso, pode-se afirmar que o parapará apresenta boa capacidade de adaptação em povoamento homogêneo independente do espaçamento inicial adotado.

A partir do oitavo ano houve maior crescimento da mortalidade, alcançando média de 34,9\% do povoamento no décimo ano nos diferentes espaçamentos. Este aumento na taxa de mortalidade se deve às características da espécie parapará, a qual demanda luz solar para seu desenvolvimento, não tolerando o sombreamento ocorrido pelo fechamento do dossel nas idades mais avançadas do povoamento (JARDIM et al., 2007).

A altura total das árvores não diferiu significativamente nos quatros espaçamentos até os dez anos de idade, constatando que o espaçamento exerce pouca influência no crescimento em altura do parapará (Figura 2A).

A altura total média do povoamento foi de 15,8 metros no sétimo ano após o plantio. O bom crescimento em altura até o sétimo ano pode ser atribuído à estratégia de crescimento dessa espécie, com alta adaptação em espaços abertos (TONINI et al., 2008). A partir do oitavo ano observa-se

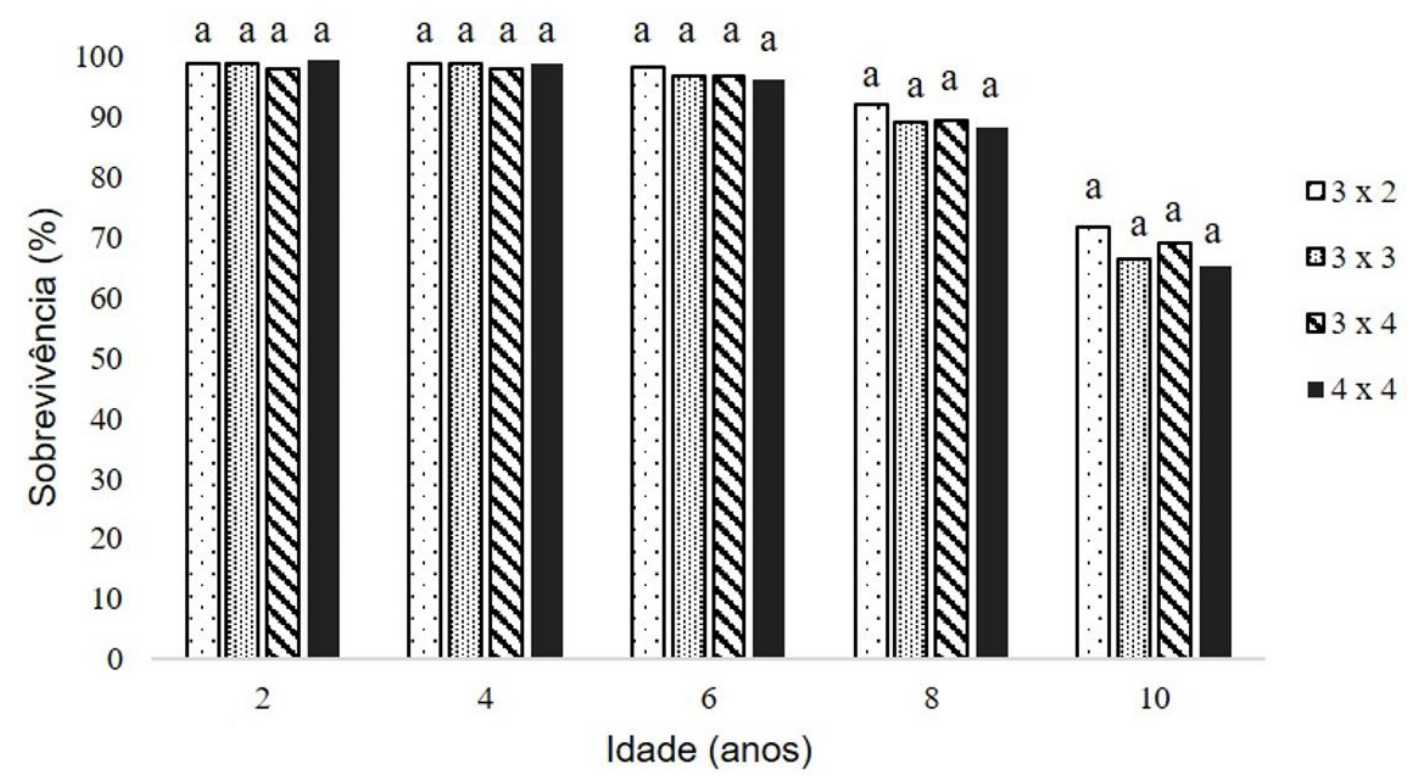

Figura 1: Sobrevivência de árvores de Jacaranda copaia em plantios homogêneos, nas idades de 2 a 10 anos, sob diferentes espaçamentos no Oeste do Estado do Pará. Letras iguais por idade não diferem estatisticamente segundo o teste de Scott Knott a 5\% de significância.

Figure 1: Survival of Jacaranda copaia trees in homogenous plantations, at ages of 2 to 10 years, under different spacings in the West of Pará State. Equivalent letters by age do not differ statistically according to the Scott Knott test at 5\% significance. 

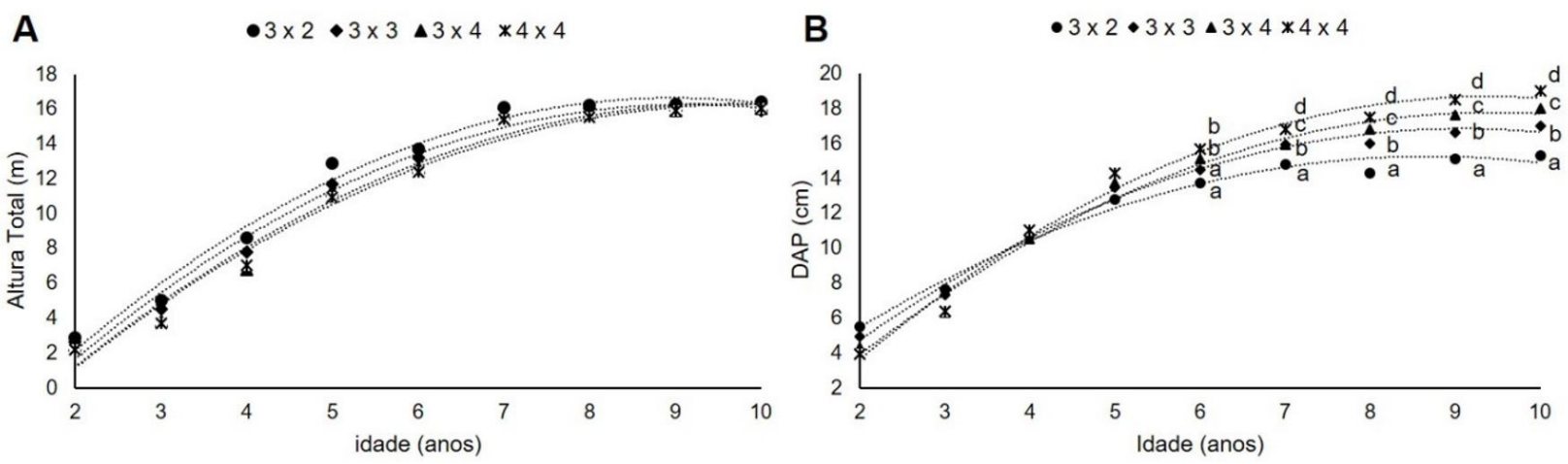

Figura 2: A. Altura total $(\mathrm{m})$ e B. Diâmetro à Altura do Peito $(\mathrm{cm})$ de árvores de Jacaranda copaia em plantios homogêneos, nas idades de 2 a 10 anos, sob diferentes espaçamentos no Oeste do Estado do Pará. Letras iguais na vertical não diferem estatisticamente segundo o teste de Scott Knott a $5 \%$ de significância.

Figure 2: A. Total height $(\mathrm{m})$ and $B$. Diameter at the height of the chest $(\mathrm{cm})$ of Jacaranda copaia trees in homogeneous plantations, in the ages of 2 to 10 years, under different spacings in the West of the State of Pará. Equal vertical letters do not differ statistically according to the Scott Knott test at $5 \%$ significance.

tendência de estagnação do crescimento em altura, explicada pela menor disponibilidade de luz para as plantas devido o adensamento das copas. Erskine et al., (2005) observaram que espécies de estágios sucessionais iniciais, com madeira de baixa densidade, como o parapará, apresentam maior crescimento quando expostas à maior radiação solar.

No que se refere ao DAP, o maior crescimento foi observado principalmente até o sétimo ano. Diferenças significativas no crescimento entre os espaçamentos foram observadas a partir do sexto ano após o plantio, sendo que os maiores valores de DAP foram observados nos espaçamentos $3 \mathrm{~m} \times 4 \mathrm{~m}$ e $4 \mathrm{~m}$ x $4 \mathrm{~m}$ em comparação aos espaçamentos $3 \mathrm{~m}$ x $2 \mathrm{~m}$ e $3 \mathrm{~m} \times 3 \mathrm{~m}$. A partir do oitavo ano, todos os espaçamentos testados influenciaram no valor de DAP, havendo uma tendência de aumento à medida que o espaço útil por planta era ampliado. Isto se deve ao fato do diâmetro ser uma das variáveis dendrométricas mais influenciadas pelo espaçamento (PELISSARI et al. 2013).

Esses resultados, onde diâmetros maiores são observados em espaçamentos mais amplos também foram encontrados nos estudos das espécies amazônicas Sclerobium paniculatum e Schizolobium parahyba var. amazonicum por Narducci (2014) e Sousa (2011) respectivamente. Esses autores alegam que povoamentos muito densos tendem naturalmente a apresentar árvores de diâmetro menor, se comparados com árvores de povoamentos mais amplos, onde a concorrência por espaço útil é menor e o crescimento em diâmetro é mais acentuado.

Com relação à área basal, houve diferenças significativas entre os espaçamentos em todas as idades analisadas, nos quais se observou que o menor espaçamento ( $3 \mathrm{~m} \times 2 \mathrm{~m}$ ) foi o tratamento que apresentou a maior área basal (Figura 3A). Os maiores valores de área basal em espaçamentos mais adensados também foi encontrado por Silva et al., (2016) e Pacheco et al, (2015). Isso se deve à influência do maior número de indivíduos por unidade de área no cálculo dessa variável.

Até a idade de sete anos os tratamentos não influenciaram o crescimento volumétrico individual do parapará, demonstrando que o arranjo espacial até essa idade não limitou a eficiência dos indivíduos na utilização dos recursos do meio. A partir do oitavo ano ocorreu diferenças estatísticas entre os espaçamentos (figura 3B), onde a volumetria individual foi mais elevada com a ampliação do espaçamento.

Aos dez anos após o plantio, a existência de competição intraespecífica no povoamento promoveu valores de volume individuais estatisticamente diferentes e o espaçamento $4 \mathrm{~m} \times 4 \mathrm{~m}$ apresentou a melhor produção madeireira de parapará $\left(0,228 \mathrm{~m}^{3} /\right.$ planta). No que se refere ao volume por árvore, observa-se que até o décimo ano após o plantio, não há uma tendência de estagnação de crescimento (figura 3B).

Quanto a produção em volume por área, observa-se que a tendência de estagnação do crescimento para volume por área também ocorrerá em idades superior a dez anos (figura 3B). O povoamento sofreu influência da densidade de plantio em todos os anos avaliados, sendo que o espaçamento mais adensado ( $3 \mathrm{~m} \times 2 \mathrm{~m})$ promoveu a maior produção volumétrica por área. No sétimo ano de 
A

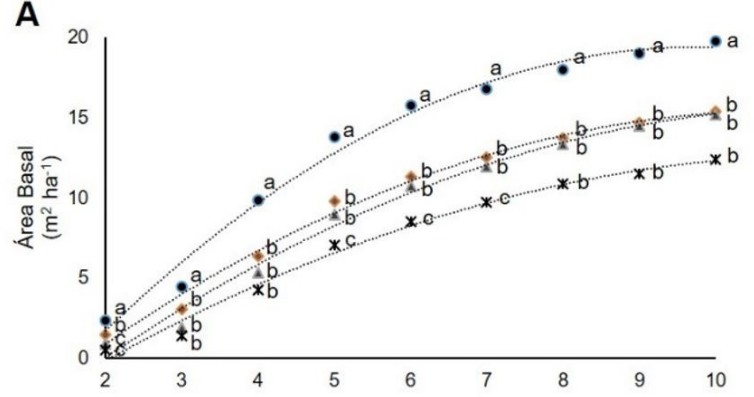

C

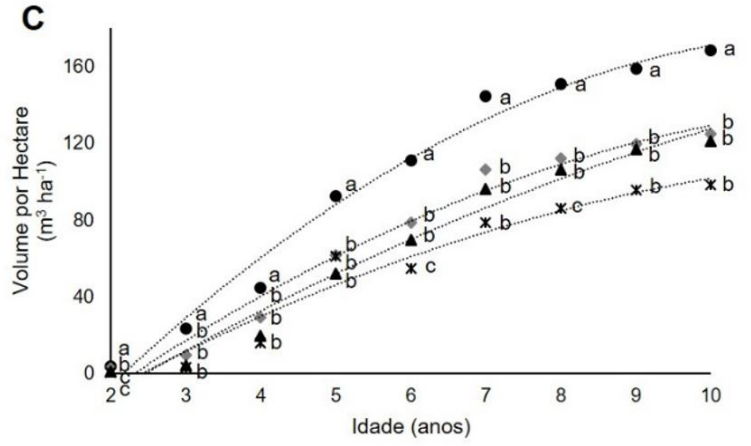

B
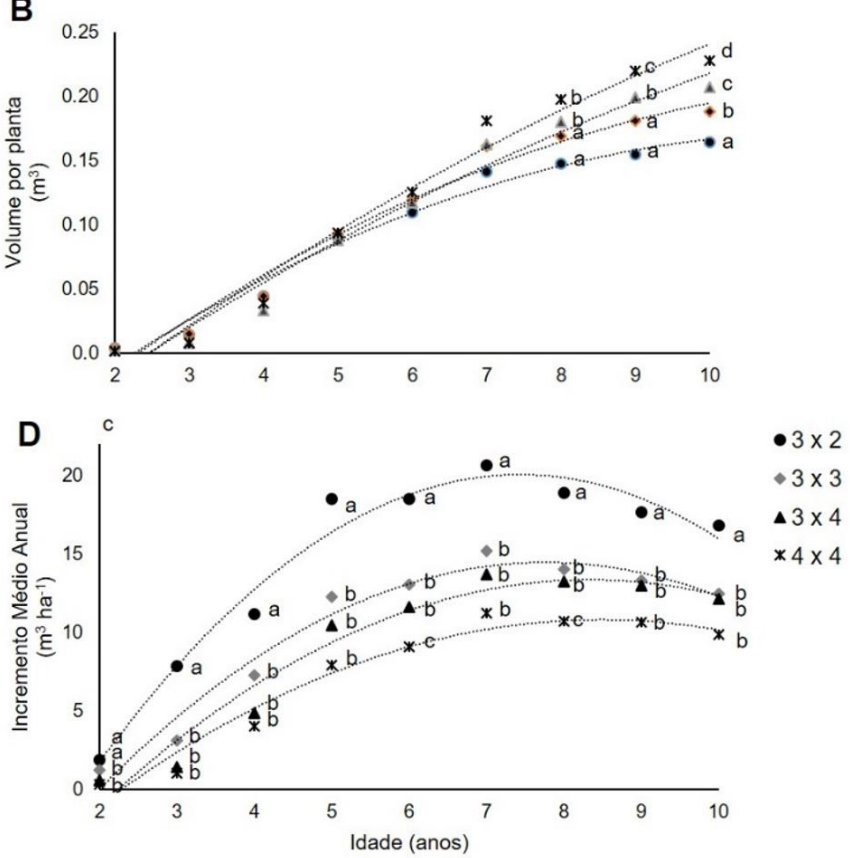

Figura 3: (A) Área Basal $\left(\mathrm{m}^{2}\right.$ ha-1); (B) volume por planta $\left(\mathrm{m}^{3}\right) ;(C)$ volume por hectare e (D) Incremento Médio Anual (m3 ha-1 ano-1) de árvores de Jacaranda copaia em plantios homogêneos, nas idades de 2 a 10 anos, sob diferentes espaçamentos no Oeste do Estado do Pará. Letras iguais na vertical diferem estatisticamente segundo o teste de Scott Knott a $5 \%$ de significância.

Figure 3: (A) Basal area $\left(\mathrm{m}^{2} \mathrm{ha}^{-1}\right)$; (B) volume per plant $\left(\mathrm{m}^{3}\right)$; (C) volume per hectare and (D) Annual average increment $\left(\mathrm{m}^{3} \mathrm{ha}^{-1}\right.$ year ${ }^{-1}$ ) of Jacaranda copaia trees in homogenous plantations, in the ages of 2 to 10 years, under different spacings in the West of the State of Pará. Vertical equal letters differ statistically according to the Scott Knott test at 5\% significance.

plantio, o volume médio para os espaçamentos $3 \mathrm{~m}$ x $3 \mathrm{~m}, 3 \mathrm{~m}$ x $4 \mathrm{~m}$ e $4 \mathrm{~m}$ x $4 \mathrm{~m}$ foi de $93,8 \mathrm{~m}^{3}$.ha $\mathrm{h}^{-1}$, foi inferior ao volume de $144,5 \mathrm{~m}^{3}$.ha ${ }^{-1}$ alcançado no espaçamento $3 \mathrm{~m} \times 2 \mathrm{~m}$.

No período de dez anos, a produção volumétrica por unidade de área foi semelhante entre os espaçamentos $3 \mathrm{~m} \times 3 \mathrm{~m}, 3 \mathrm{~m}$ x $4 \mathrm{~m}$ e $4 \mathrm{~m} \times 4 \mathrm{~m}$, com exceção dos anos 6 e 8, em que a produção foi significativamente menor sob densidade de plantio $4 \mathrm{~m} \times 4 \mathrm{~m}$. A maior produção volumétrica por área no menor espaçamento se deve ao fato desta variável estar diretamente relacionada com a sobrevivência, crescimento em altura e diâmetro dos indivíduos, bem como o número de indivíduos por unidade de área.

Diferenças significativas entre os espaçamentos foram observadas para incremento médio anual, sendo que os espaçamentos mais adensados apresentaram valores mais elevados de IMA, o que também foi observado por Pereira (2015), trabalhando com espécies do gênero Eucalyptus. Nota-se na Figura 3D uma curva de tendência na forma de parábola, com um período de crescimento bem definido até o sétimo ano, onde o IMA no espaçamento $3 \mathrm{~m} \times 2 \mathrm{~m}$ foi de $20,65 \mathrm{~m}^{3}$.ha-1, seguido de gradual redução nas idades adiantes. No oitavo ano o IMA reduziu 1,85 $\mathrm{m}^{3} \cdot \mathrm{ha}^{-1}$.

O IMA representa a melhor estimativa da taxa máxima de crescimento que pode ser mantida de forma contínua por uma determinada espécie em um determinado sítio (SMITH et al., 1997). A redução do IMA implica dizer que a partir daquela idade o povoamento diminuiu a velocidade de crescimento, portanto é o momento ideal de intervir no povoamento, seja com a exploração ou com desbaste. A partir dos resultados observados na Figura 3D, o sétimo ano é a idade adequada para intervenção silvicultural no povoamento estudado.

Para a implantação de povoamentos homogêneos de Jacaranda copaia pode-se adotar inicialmente os espaçamentos $3 \mathrm{~m} \times 3 \mathrm{~m}$ ou $3 \mathrm{~m} \times 4 \mathrm{~m}$, desde que a localidade apresente características ambientais semelhantes às do presente estudo. Esses espaçamentos são sugeridos considerando que a destinação da madeira dessa espécie será para indústria de laminados e marcenaria, a qual não exige árvores de diâmetros muito elevados como obtido no espaçamento $4 \mathrm{~m}$ x $4 \mathrm{~m}$. O espaçamento $3 \mathrm{~m}$ x $2 \mathrm{~m}$ implica diretamente no manejo e colheita do povoamento devido o adensamento das copas. 


\section{CONCLUSÃO}

O povoamento de Jacaranda copaia apresentou elevada sobrevivência durante o período estudado. Considerando o uso da madeira de Jacaranda copaia e o desempenho silvicultural apresentado nesse estudo, pode-se adotar espaçamentos $3 \mathrm{~m}$ x $3 \mathrm{~m}$ ou $3 \mathrm{~m}$ x $4 \mathrm{~m}$ na implantação de povoamentos homogêneos dessa espécie. E o sétimo ano é a idade adequada para intervenção silvicultural no povoamento, independente do espaçamento adotado.

\section{REFERENCIAS BIBLIOGRÁFICAS}

CARVALHO, P. E. R. C. Comparação de espécies nativas, em plantio em linha, em capoeira, na região de Irati - PR. Boletim de Pesquisa Florestal, Colombo, n.5, p.53-68, dez. 1982.

ERSKINE, P.D., LAMB, D., BORSCHMANN, G. Growth performance and 431 management of a mixed rainforest tree plantation. New Forests. v. 29. p. 117-134. 2005.

FERREIRA, D. F. Sisvar: a guide for its bootstrap procedures in multiple comparisons. Ciência e Agrotecnolgia, v. 38. p. 109-112. 2014.

IPEF - INSTITUTO DE PESQUISAS E ESTUDOS FLORESTAIS. Identificação de espécies florestais: Jacaranda copaia. 2016. Disponível em: < http://www.ipef.br/identificacao/nativas/detalhes.asp?codigo=64 >. Acesso em 20 de agosto de 2016.

JARDIM, F. C. DA S.; SERRÃO, D. S.; NEMER, T. C. Efeito de diferentes tamanhos de clareiras, sobre o crescimento e a mortalidade de espécies arbóreas, em Moju-PA. Revista Acta Amazonica., Manaus, v. 37, n. 1, p. 37 - 47 , 2007.

NARDUCCI, T. S. Recuperação de Áreas De Reserva Legal: Influência da densidade nos indicadores ambientais do plantio Sclerolobium paniculatum 2017. 77 p. Dissertação (Mestrado em Recursos Florestais) - Universidade Federal do Pará, Belém, 2017

NASCIMENTO, D. F. DO; LELES, P. S. DOS S.; OLIVEIRA NETO, S. N. DE; MOREIRA, R. T. S; ALONSO, J. M. Crescimento Inicial de Seis Espécies Florestais em Diferentes Espaçamentos. Revista Cerne. v. 18, n. 1, p. 159-165. 2012.

OLIVEIRA JÚNIOR, R. C.; CORREA, J. R. V. Caracterização dos solos do Município de Belterra, Estado do Pará. Belém: Embrapa Amazônia Orientall, 2001. 39 p. (Embrapa Amazônia Oriental, Documentos, 88). Documentos. EMBRAPA Amazônia

PACHECO, J. M.; FIGUEIREDO FILHO, A.; DIAS, A. N.; MACHADO, S. A.; LIMA, R. Efeito da densidade inicial no crescimento de Pinus taeda L. na região Centro Sul do Paraná. Revista Scientia Forestais. v. 43, n. 106, p. 353-365. 2015.

PELISSARI A. L, CALDEIRA S. F. DRESCHER, R. Desenvolvimento quantitativo e qualitativo de Tectona grandis L.f. em Mato Grosso. Revista Floresta e Ambiente. v. 20. p.371-383, 2013.

PEREIRA, D. G. L. Influência do espaçamento de plantio em povoamento de clones híbridos de Eucalyptus spp. no Estado do Pará. 2015. 22 p. Monografia (Graduação em Engenharia Florestal) - Universidade Federal do Paraná, Curitiba, 2015.

SCHNEIDER, P. R.; FINGER, A. G.; SCHNEIDER, P. S. P.; FLEIG, F. D.; CUNHA, T. A. Influência do espaçamento no autodesbaste de povoamento monoclonal de Eucalyptus saligna Smith. Revista Ciência Florestal, Santa Maria, v. 25, n. 1, p. 119-126, 2015.

SMITH, D.M.; LARSON, B, C.; KELTY, M, J.; ASHTON, P.MARK. The practice of silviculture. Applied forest ecology. New York: J.Wiley, 1997, 517 p.

SOUSA, V. G. Comportamento silvicultural e dinâmica de serapilheira em plantios de duas espécies florestais na Amazônia Oriental Brasileira. 2011. 111 p. Dissertação (Mestrado em Recursos Florestais) Universidade Federal do Pará, Curitiba, 2011. 
SILVA, J. M. L. DA; SANTOS, P. L.; LIMA. A. A. C.; MARTINS, J. S.; RODRIGUES, T. E.; VALENTE, M. A.; RÊGO, R. S.; CARDOSO JUNIOR, E. Q. Levantamento semidetalhado dos solos do Campo Experimental de Belterra - Estado do Pará. Belém: Embrapa Amazônia Oriental, 2000. 30 p. (Embrapa Amazônia Oriental, Documentos, 57).

SILVEIRA, R. DA. Avaliação econômica da produção de madeira de paricá (Schizolobium amazonicum Huber ex Ducke) sob diferentes espaçamentos de plantio. Vitória: Universidade, 2014. 82 p. Dissertação de mestrado.

VENTURIN, N.; CARLOS, L. SOUZA, P. A DE; MACEDO, R. L. G. VENTURIN, R. P.; HIGASHIKAWA, E. M. Desempenho Silvicultural de Acrocarpus fraxinifolius Wight em função de diferentes espaçamentos e idades. Revista Cerne, Lavras, v. 20 n. 4, p. 629-636. 2014.

TONINI, H.; ARCO-VERDE; M. F; SÁ, S. P. P. Dendrometria de espécies nativas em plantios homogêneos no Estado de Roraima - Andiroba (Carapa guianensis Aubl), Castanha-do-Brasil (Bertholletia excelsa Bonpl.), Ipê-roxo (Tabebuia avellanedae Lorentz ex Griseb) e Jatobá (Hymenaea courbaril L.). Revista Acta Amazonica, Manaus, v. 35. p. 353 - 362, 2005.

TONINI, H., OLIVEIRA jr, M. M. C.; SCHWENGBER, D. Crescimento de espécies nativas da Amazônia submetidas ao plantio no estado de Roraima. Revista Ciência Florestal, Santa Maria, v. 2, p. 151-158. 2008. 